\title{
Comprehensive approach to peritoneal dialysis-related peritonitis by enteric microorganisms: comparison between single organism and polymicrobial infections
}

\author{
Roi Ribera-Sánchez ${ }^{1}$, Miguel Pérez-Fontán ${ }^{1,2}$, Antía López-Iglesias ${ }^{1}$, Alba García- \\ Enríquez ${ }^{1}$ and Ana Rodríguez-Carmona ${ }^{1}$ \\ ${ }^{1}$ Division of Nephrology, University Hospital A Coruña, Spain \\ ${ }^{2}$ Department of Medicine, Health Sciences Faculty, University of A Coruña, Spain
}

\begin{abstract}
Background: Peritoneal infections of enteric origin (EntP) have been classically investigated using partial strategies, focused on particular subgroups of microorganisms. A more comprehensive approach may facilitate the definition of the nomenclature and clinical presentation of these infections.

Objectives: To investigate the clinical presentation and outcomes of a full spectrum of EntP, with a particular interest in the comparison between single-organism and polymicrobial infections.

Method: Following an observational design, we investigated 165 single-organism and 83 polymicrobial peritonitis episodes with isolation of at least 1 enteric bacteria (Enterobacteriaceae, Enterococcus spp. and/or intestinal anaerobics). We compared the risk of treatment failure for these 2 types of infection and explored the significance of the isolation of specific microorganisms and of their antibacterial susceptibility patterns.

Results: Polymicrobial EntP was associated with higher rates of hospitalization, more changes to initial antibiotic therapy, more surgical explorations, and higher mortality and treatment failure rates than monobacterial EntP. However, stratified and multivariate analyses revealed that the burden of these differences rested on the isolation of intestinal anaerobics (odds ratio [OR] 12.05, 95\% confidence interval [CI] 2.53-31.09, $p<0.001$ ) and/or Enterococcus faecium (OR 3.37, 95\% CI 1.02-11.30, $p=0.046$ ), while other polymicrobial infections were more comparable with single-organism peritonitis, except for even higher mortality rates in the former group. Lower antibiotic susceptibility of the isolations (OR $1.18,95 \%$ CI $0.51-2.70, p=0.70)$ did not perform as a predictor of treatment failure.

Conclusion: A comprehensive approach to peritoneal infections by intestinal microorganisms may provide a focused perspective of the clinical presentation and outcomes of these complications of peritoneal dialysis.
\end{abstract}

\section{Keywords}

Peritoneal infection; Enterobacteriaceae; Anaerobic; Enterococcus; Mortality; Treatment failure 
Peritoneal infections with an enteric origin (EntP) represent one of the most feared complications of peritoneal dialysis (PD), due both to the difficulties of determining an accurate diagnosis and management, and to their significant complication rates, including mortality and PD technique failure $(1,2)$. One important limitation at the time of approaching EntP is the lack of a standardized definition of these infections. In fact, current International Society for Peritoneal Dialysis (ISPD) guidelines do not even attempt a comprehensive approach to the diagnosis and management of EntP (3). Overall, only peritoneal infections caused by at least 2 different microorganisms of intestinal origin or 1 enteric anaerobic bacteria are universally recognized as EntP (3). Similar considerations may apply for peritonitis caused by 1 single enteric bacterium (including Enterobacteriaceae and Enterococcus spp.), but direct categorization of these infections as EntP may be more controversial because, in these cases, alternative pathways of contamination are feasible. The convenience of categorizing monobacterial together with polymicrobial peritonitis by intestinal microorganisms as EntP depends largely on the compared clinical presentation, management, and outcomes of these infections. However, there are surprisingly few studies addressing this question (4,5). In particular, the influence of clinical and microbiologic factors, including the etiologic agents and their antibiotic susceptibility patterns, on the presentation of EntP has not been sufficiently investigated.

We have undertaken an observational study, based on a comprehensive approach to the clinical presentation and outcomes of peritoneal infections caused by a full spectrum of microorganisms of presumed enteric origin. Our main objective was to compare the outcomes of single-organism and polymicrobial peritonitis, paying particular attention to the significance of the isolation of specific microorganisms.

\section{METHOD}

\section{GENERAL DESIGN}

Following an observational, retrospective design, we analyzed the clinical presentation of peritoneal infections with a presence of at least 1 microorganism of presumed enteric origin, diagnosed in our center between January 1990 and December 2016. Primary objectives of our analysis included a comparison of the clinical presentation of monobacterial (MEntP) and polymicrobial (PEntP) infections, as well as exploring potential markers of a complicated clinical course in these 2 groups of infections.

The study was carried out in a tertiary university hospital attending to 100 to 120 patients on PD per year, during the whole study period. The main study variable was the single-organism or polymicrobial character of peritoneal infections by intestinal microorganisms. The main outcome was a composite variable reflecting treatment failure (see below). We also analyzed a wide set of control variables with a potential impact on the presentation or outcome of these infections.

The present study followed the ethical principles for medical research included in the declaration of Helsinki, and complied with the requirements of our center for observational, retrospective studies. We requested and obtained oral consent for the study from all patients available at the initiation of the study. 
The main subjects of our analysis were episodes of peritoneal infection with the presence of at least 1 intestinal microorganism recorded during the aforementioned period, including:

1) Monobacterial infections caused by microorganisms with a usual enteric origin, including Enterococcus spp., Enterobacteriaceae, and intestinal anaerobics.

2) PEntP, defined by the isolation of 2 or more microorganisms, at least 1 of them having a presumed enteric origin.

We considered only cases with complete clinical records, including a follow-up until the end of the study period or PD drop-out for any reason (switch to hemodialysis, kidney transplant, or death).

We excluded from analysis the following instances:

- Infections with a rampant surgical background, defined by an immediate (first 24 hours) diagnosis and/or outcome. Consequently, we excluded the so-called abdominal catastrophes (mesenteric thrombosis, overt intestinal perforation, and other surgical processes undergoing a straight diagnosis and management)

- PEntP with a primary isolation of yeasts or filamentous fungi, due to their specific clinical presentation and management. On the other hand, we considered cases with a secondary isolation (reinfection) of these microorganisms

- MEntP with simultaneous catheter exit-site or tunnel infection by the same etiologic agent (catheterdependent infections)

- Infections by microorganisms with a possible, but not unequivocal, enteric origin, including nonfermenting gram-negative bacteria or Streptococcus spp.

- Relapses of EntP, which were categorized as a part of the original episode

\section{STUDY VARIABLES}

The main study variable was the single-organism or polymicrobial nature of EntP. Secondary study variables included the isolation of Escherichia coli, Enterococcus spp., or anaerobics as etiologic agents, as well as the antibacterial susceptibility patterns of the isolations. The latter were categorized for analysis according to the minimal inhibitory concentrations (MIC) for vancomycin (Enterococcus spp.), cefotaxime, ceftazidime, imipenem-cilastatin, gentamycin, and ciprofloxacin (gram-negative bacteria). We categorized the antibiotic resistance pattern of gram-negatives as low (susceptible) when the isolations was resistant to, at most, 1 of the 5 aforementioned antibiotics; intermediate susceptibility was defined by resistance to 2 to 3 antibacterials. Multiresistance was defined by non-susceptbility to at least 4 of the tested antibacterials. Regarding enterococci, we observed no single instance of overt resistance to vancomycin, and we categorized as intermediate resistance a MIC $>2 \mathrm{mcg} / \mathrm{mL}$. The antibiotic susceptibility patterns of anaerobic bacteria were not routinely tested. In the case of PEntP, overall antibacterial susceptibility was categorized according to the less favorable pattern of resistance.

The main outcome variable was treatment failure, defined by at least 1 of the following 3: peritoneal catheter removal, transfer to hemodialysis for at least 3 months after the infection, or death related to peritonitis (demise for any reason during hospital admission or within 30 days after initiation of the episode). Secondary outcome variables included: hospitalization, number of days with a documented peritoneal inflammatory response (delay to the first dialysate leukocyte count $<100 / \mathrm{mm} 3$ or to catheter removal), changes to the initial empiric antibiotic therapy, abdominal surgical exploration, and relapse and reinfection, according to the ISPD criteria (3). 
The main control variables included age, gender, presence of diabetes, time on PD at the time of the infection, number of major comorbidities, previous immunosuppressive therapy, malnutrition (subjective global assessment), and the latest plasma albumin level before the episode of infection (median 2 months, range $0-4)$.

\section{DEFINITION OF VARIABLES AND CLINICAL PROCEDURES}

The general diagnosis of peritoneal infection, as well as the nomenclature related to its outcomes, complied with the ISPD standards (3). In our center, hospital admission for peritoneal infection is indicated for 3 main reasons: high-risk patients, complicated infections (aggressive clinical presentation, refractoriness to appropriate antibiotic therapy), or isolation of microorganisms demanding in-center management (e.g. yeasts).

In our center, the protocol for initial treatment of peritoneal infections has been modified over time, according to variations in the antibiotic susceptibility patterns of the isolations obtained. Between 1990 and 2007, initial therapy was based on intraperitoneal ciprofloxacin. After a progressive increase in the rates of resistance of coagulase-negative staphylococci (but not of gram-negative bacteria) in 2007, we moved to a new schedule based on intravenous vancomycin and intraperitoneal cefotaxime, which still stands. After the initial diagnosis of peritonitis, we perform clinical, cytologic, and bacteriologic controls every 48 hours until full remission of the infection. Antibiotic therapy is adapted to the susceptibility patterns of the isolations and maintained for a minimum of 2 weeks, following the ISPD recommendations (3). Usual changes to the initial treatment after isolation of gram-negatives include addition of a second antibacterial (more commonly an aminoglycoside) or switch to a carbapenem, on an individual basis. Antibiotic susceptibility is tested using a standard MIC method.

Between 1990 and 2010 the indication for abdominal imaging was individualized. Since 2010, we perform routine computed tomography (CT) scans on all patients with peritoneal infection and isolation of at least 1 enteric microorganism. The indications for exploratory laparoscopy/laparotomy remain individualized.

\section{DATA ANALYSIS AND STATISTICS}

Numeric variables are presented as mean values (standard deviation), and categorical variables as the number of cases (\%). Univariate comparisons between MEntP and PEntP were produced using 2-tailed Student's $t$-test, ANOVA (Scheffé) (numeric) and $\chi^{2}$ distribution (categorized). We first compared the demographic, clinical, and evolutionary patterns associated with MEntP and PEntP. We performed subanalyses to disclose the impact of the specific isolations of Escherichia coli, Enterococcus spp., and anaerobic bacteria. Finally, we investigated the significance of the antibacterial susceptibility patterns for the outcome of the infections.

We applied stepwise logistic regression analysis to investigate predictors of the main outcome variable (treatment failure). A preliminary, exploratory analysis identified gender, plasma albumin, and time on dialysis at the moment of the infection as variables with a potential association with the main outcome. Subsequently, we investigated the adjusted effect of the following primary and secondary study variables on the risk of treatment failure: single-organism versus polymicrobial infection, isolation of specific bacteria (Escherichia coli, Enterococcus spp., and anaerobics), and the antibiotic susceptibility patterns of the isolations. We managed the latter variable binarily (higher/lower susceptibility) due to a low proportion of highly multiresistant strains (see Results).

We performed statistical analysis with the help of the SPSS 19.0 software (SPSS, Chicago, IL, USA). 


\section{RESULTS}

\section{OVERVIEW}

We recorded 1,066 episodes of peritoneal infection during the study period, including $800(75.0 \%)$ caused by a single microorganism, $153(14.4 \%)$ by 2 or more microorganisms, and $113(10.6 \%)$ culturenegative cases. The series included 270 episodes of peritoneal infection with isolation of enteric microorganisms. Of these, we excluded from analysis 22 episodes due to incomplete clinical records $(n=$ $5)$, simultaneous catheter exit-site infection by the same bacteria $(n=9)$, rampant surgical nature of the event $(n=6)$, or primary isolation of yeasts in a setting of PEntP $(n=2)$. In total, we analyzed 165 episodes of MEntP and 83 episodes of PEntP.

The etiologic agents of infection are shown in Table 1, while Table 2 compares the demographic, clinical, and microbiologic characteristics of MEntP and PEntP at presentation. Patients suffering PEntP were marginally older, without other apparent differences between the groups.

TABLE 1. Etiologic Agents of Peritoneal Infection

\begin{tabular}{lcc}
\hline & MEntP $n=165$ & PEntP $n=83$ \\
\hline & $33(20.0)$ & $29(34.9)$ \\
Enterococcus spp. & $26(15.8)$ & $17(20.5)^{\mathrm{a}}$ \\
E. faecalis & $5(3.0)$ & $11(13.3)^{\mathrm{a}}$ \\
E. faecium & $2(1.2)$ & $2(2.4)$ \\
Other & $59(35.7)$ & $46(55.4)$ \\
Escherichia coli & $28(17.0)$ & $11(13.3)$ \\
Klebsiella spp. & $14(8.5)$ & $3(3.6)$ \\
Serratia spp. & $13(7.9)$ & $6(7.2)$ \\
Enterobacter spp. & $5(3.0)$ & $6(7.2)$ \\
Proteus spp. & $2(1.2)$ & $11(13.3)$ \\
Morganella spp. & $3(1.8)$ & $4(4.8)$ \\
Citrobacter spp. & $5(3.0)$ & $3(3.6)$ \\
Other enteric gram-negatives & $3(1.8)^{\mathrm{b}}$ & $20(24.1)$ \\
Enteric anaerobics & - & $5(6.1)$ \\
Non-fermenting gram-negatives & - & $2(2.4)$ \\
Other non-enteric gram-negatives & - & $31(37.3)$ \\
Streptococcus spp. & - & $15(18.1)$ \\
Staphylococcus spp. & - & $1(1.2)$ \\
Other non-enteric gram-positives & & \\
&
\end{tabular}

$\mathrm{MEntP}=$ single-organism enteric peritonitis; $\mathrm{PEnt} \mathrm{P}=$ polymicrobial enteric peritonitis.

Figures denote number of episodes (\%).

${ }^{a}$ E. faecalis and E. faecium coexisted in 1 case.

b Clostridium perfringens, Bacteroides fragilis and unidentified anaerobic gram-negative. 
TABLE 2. Demographic and Microbiologic Differences According to Study Group

\begin{tabular}{|c|c|c|c|}
\hline & MEntP & PEntP & $p$ value \\
\hline $\mathrm{N}$ & 165 & 83 & \\
\hline Age (years) & $62.9(14.2)$ & $66.1(11.6)$ & 0.058 \\
\hline Males/females (\%) & $61.8 / 38.2$ & $55.4 / 44.6$ & 0.31 \\
\hline Time on PD (months) & $24.0(19.2)$ & $25.8(24.2)$ & 0.58 \\
\hline Modality of PD (CAPD/automated PD) (\%) & $67.3 / 32.7$ & $67.5 / 32.5$ & 0.69 \\
\hline Diabetes $(\%)$ & 31.5 & 25.3 & 0.35 \\
\hline Comorbidity score & $2.91(2.51)$ & $2.44(2.27)$ & 0.14 \\
\hline Previous kidney transplant (\%) & 6.7 & 3.6 & 0.39 \\
\hline Previous/current immunosuppressives (\%) & 12.1 & 7.3 & 0.28 \\
\hline Malnutrition (\%) & 14.5 & 4.8 & 0.097 \\
\hline Plasma albumin $(\mathrm{g} / \mathrm{L})$ & $36.1(5.5)$ & $36.8(5.4)$ & 0.80 \\
\hline Antibiotic resistance patterns $(\%)^{\mathrm{a}}$ & 75.3 & 67.1 & 0.24 \\
\hline Susceptible & 23.4 & 28.6 & \\
\hline Intermediate resistance & 1.3 & 4.3 & \\
\hline Multiresistance & & & \\
\hline
\end{tabular}

$\mathrm{MEntP}=$ single-organism enteric peritonitis; $\mathrm{PEntP}=$ polymicrobial enteric peritonitis; $\mathrm{PD}=$ peritoneal dialysis; $\mathrm{CAPD}=$ continuous ambulatory $\mathrm{PD}$.

Figures denote mean values (standard deviation)(numeric variables) or \% (categorical variables).

${ }^{a}$ Enterococcus spp. and enteric gram-negatives.

The MIC90 values for the isolated gram-negative bacteria were $32 \mathrm{mcg} / \mathrm{mL}$ (cefotaxime), $4 \mathrm{mcg} / \mathrm{mL}$ (ceftazidime), $4 \mathrm{mcg} / \mathrm{mL}$ (imipenem-cilastatin), $1 \mathrm{mcg} / \mathrm{mL}$ (ciprofloxacin) and $8 \mathrm{mcg} / \mathrm{mL}$ (gentamycin), respectively. Vancomycin MIC90 for enterococci was $2 \mathrm{mcg} / \mathrm{mL}$. We recorded 3 instances of extendedspectrum beta-lactamase-producing Enterobacteriaceae, and no further analysis was dedicated to this particular subset.

\section{COMPARED OUTCOMES OF MEnTP AND PEntP}

Table 3 compares the clinical presentation and outcomes of MEntP and PEntP. Some of the differential features could be, at least partly, a consequence of the concerns raised by the isolation of polymicrobial flora. Thus, PEntP was associated with more hospitalizations, longer in-hospital stays, and more frequent changes to the initial antibiotic therapy. In addition, surgical exploration was more frequently indicated in the presence of PEntP. Most importantly, mortality and treatment failure rates were clearly higher in these cases. On the other hand, relapses were more frequent in MEntP. 
TABLE 3Clinical Presentation and Outcomes According to Type of Infection

\begin{tabular}{|c|c|c|c|}
\hline & MEntP & PEntP & $p$ value \\
\hline$N$ & 165 & 83 & \\
\hline Hospital admission (\%) & 30.3 & 48.2 & 0.012 \\
\hline No. of days of admission & $5.6(13.5)$ & $15.2(30.7)$ & 0.008 \\
\hline No. of days with peritoneal inflammation & $6.0(3.7)$ & $5.7(3.3)$ & 0.64 \\
\hline Peritoneal cell count at baseline (per mm3) & $3,566(6,303)$ & $2,841(2,883)$ & 0.63 \\
\hline$\%$ of neutrophils at baseline & $76.9(13.2)$ & $77.1(16.1)$ & 0.93 \\
\hline Changes to initial antibacterial therapy (\%) & & & 0.001 \\
\hline Did not change & 55.8 & 36.8 & \\
\hline Torpid/refractory infection & 21.1 & 27.6 & \\
\hline In vitro resistance & 17.6 & 15.8 & \\
\hline Late isolations & 2.4 & 17.1 & \\
\hline Systemic compromise & 1.2 & 1.3 & \\
\hline Relapse under treatment & 1.8 & 1.3 & \\
\hline Peritoneal catheter removed (\%) & 17.0 & 25.3 & 0.15 \\
\hline Relapse (\%) & 18.8 & 7.2 & 0.048 \\
\hline Reinfection $(\%)$ & 4.8 & 1.2 & 0.21 \\
\hline Other bacteria & 2.4 & 2.4 & \\
\hline \multicolumn{4}{|l|}{ Yeasts } \\
\hline Surgical exploration (\%) & 3.0 & 18.0 & 0.001 \\
\hline Final outcome $(\%)$ & 87.3 & 66.3 & 0.001 \\
\hline PD continued for at least 3 months & 5.4 & 13.3 & \\
\hline Drop-out to hemodialysis & 7.3 & 20.5 & \\
\hline Death & & & \\
\hline Treatment failure $^{\mathrm{a}}(\%)$ & 20.0 & 37.3 & 0.003 \\
\hline
\end{tabular}

$\mathrm{MEntP}=$ single-organism enteric peritonitis; $\mathrm{PEntP}=$ polymicrobial enteric peritonitis $; \mathrm{PD}$ $=$ peritoneal dialysis.

Figures denote mean values (standard deviation)(numeric variables) or \% (categorical variables).

${ }^{a}$ Demise and/or drop-out to hemodialysis and/or catheter removed.

Overall, surgical exploration was undertaken in 21 patients. In 8 cases ( 3 MEntP and 5 PEntP), no evident cause for the infection was identified ("white" laparotomies). Diagnoses during the remaining explorations included acute diverticulitis $(n=4)$, bowel perforation in a setting of intestinal ischemia $(n=$ $3)$, acute cholecystitis $(n=3)$, acute appendicitis $(n=2)$, and acute pancreatitis $(n=1)$. Surgical exploration was more frequently performed in PEntP than in MentP (Table 3), as well as when anaerobic bacteria were isolated $(47.8 \%$ vs $4.4 \%, \mathrm{p}<0.001)$. Remarkably, 3 "white" laparotomies occurred in patients suffering PEntP with the presence of anaerobics.

On univariate analysis, the incidence of treatment failure was $29.8 \%$ when Escherichia coli was isolated, as compared with $27.1 \%$ in the opposite case $(p=0.64)$. The presence of Enterococcus spp. overall did not result in different rates of this outcome $(28.3 \%$ vs $28.1 \%, \mathrm{p}=0.98)$. On the other hand, isolation of Enterococcus faecium portended a complicated clinical course $(50.0 \%$ vs $26.4 \%, \mathrm{p}=0.024)$. The most consistent univariate predictor of treatment failure was isolation of anaerobic bacteria $(78.9 \%$ vs $24.2 \%, \mathrm{p}<0.001)$.

Table 4 displays the clinical presentation and outcomes of MEntP and PEntP, after stratification for the presence or absence of anaerobic bacteria in the case of PEntP. Under these conditions, the outcomes of MEntP and PEntP without anaerobics were more comparable, although some differences persisted, including a higher rate of mortality in the latter group. 
TABLE 4. Clinical Presentation and Outcomes According to Type of Infection and Presence or Absence of Anaerobicsa

\begin{tabular}{|c|c|c|c|}
\hline & MEntP, no anaerobics & PEntP, no anaerobics & PEntP with anaerobics \\
\hline$N$ & 162 & 63 & 20 \\
\hline Hospital admission (\%) & 28.4 & 39.1 & 95.0 \\
\hline No. of days of admission & $5.1(11.3)$ & $7.3(18.6)$ & $42.5(44.7) \mathrm{c}$ \\
\hline No. of days with peritoneal inflammation & $6.0(3.7)$ & $5.6(3.4)$ & $6.3(3.4)$ \\
\hline Baseline peritoneal cell count (per $\mathrm{mm}^{3}$ ) & $3556(6297)$ & $2812(2542)$ & $2977(3771)$ \\
\hline$\%$ of neutrophils at baseline & $76.8(13.2)$ & $77.4(16.3)$ & $75.5(15.8)$ \\
\hline \multicolumn{4}{|l|}{ Changes to initial antibacterial therapy (\%) } \\
\hline Did not change & 56.8 & $41.4^{\mathrm{d}}$ & $10.0^{\mathrm{c}}$ \\
\hline Torpid/Refractory infection & 21.0 & 24.1 & 55.0 \\
\hline In vitro resistance & 17.3 & 19.0 & 5.0 \\
\hline Late isolations & 1.9 & 13.8 & 25.0 \\
\hline Systemic compromise & 1.2 & 1.7 & 0 \\
\hline Relapse under treatment & 1.8 & 0 & 5.0 \\
\hline Peritoneal catheter removed (\%) & 17.0 & 14.1 & $65.0^{\mathrm{c}}$ \\
\hline Relapse (\%) & 18.8 & $6.3^{\mathrm{e}}$ & 10.0 \\
\hline \multicolumn{4}{|l|}{ Reinfection (\%) } \\
\hline Other bacteria & 4.8 & 0 & 5.0 \\
\hline Yeasts & 2.4 & 3.2 & 0 \\
\hline Surgical exploration (\%) & 3.0 & 6.3 & $55.0 \mathrm{c}$ \\
\hline \multicolumn{4}{|l|}{ Final outcome $(\%)$} \\
\hline PD continued for $>3$ months & 88.3 & 76.5 & $30.0^{\mathrm{c}}$ \\
\hline Drop-out to hemodialysis & 5.5 & 6.3 & $35.0^{\mathrm{c}}$ \\
\hline Death & 6.2 & $17.2^{\mathrm{f}}$ & $35.0^{\mathrm{c}}$ \\
\hline Treatment failureb (\%) & 19.8 & 25.3 & $80.0^{\mathrm{c}}$ \\
\hline
\end{tabular}

$\mathrm{MEntP}=$ single-organism enteric peritonitis; $\mathrm{PEntP}=$ polymicrobial enteric peritonitis; $\mathrm{PD}=$ peritoneal dialysis . Figures denote mean values (standard deviation)(numeric variables) or \% (categorical variables).

${ }^{\text {a }}$ Data for monobacterial anaerobic infections $(n=3)$ not presented.

${ }^{\mathrm{b}}$ Demise and/or drop-out to hemodialysis and/or catheter removed.

${ }^{c} p<0.001$ vs any other group.

d $p=0.012$ vs monobacterial.

e $p=0.058$ vs monobacterial.

${ }^{\mathrm{f}} p=0.022$ vs monobacterial.

Other differences not significant.

\section{MULTIVARIATE ANALYSIS}

Logistic regression analysis (Table 5) confirmed the isolation of anaerobic bacteria as the most consistent predictor of treatment failure. Our data also indicated that isolation of Enterococcus faecium may portend a similar prognosis. On the other hand, the influence of the number of isolations or the antibiotic resistance patterns of the causing microorganisms did not bear an independent impact on the general prognosis of the infections although, in the former case, data suggested a minor trend to a higher risk for PEntP than for MEntP. A secondary analysis did not disclose a different outcome in PEntP with 2 $(n=59)$ versus 3 or more isolations $(n=24)$, after controlling for the presence of anaerobics and Enterococcus faecium (odds ratio [OR] 0.96, 95\% confidence interval [CI] $0.30-3.07, \mathrm{p}=0.96$ ).

An analysis specifically oriented to the risk of peritonitis-related mortality disclosed that after controlling for other independent predictors of this outcome (age, plasma albumin, presence of anaerobics, and time on PD at infection), PEntP was associated with a higher risk of this event than MEntP (OR 3.38, 95\% CI $1.27-9.03, \mathrm{p}=0.008$ ). 
TABLE 5. Predictors of Treatment Failure: Multivariate, Logistic Regression Analysis

\begin{tabular}{lccc}
\hline \multicolumn{1}{c}{ Best model } & OR & $95 \%$ CI & $p$ value \\
\hline & & & \\
Time on PD (per month) & 1.04 & $1.03-1.06$ & 0.001 \\
Plasma albumin (per 1 g/L) & 0.92 & $0.86-0.99$ & 0.017 \\
Anaerobics isolated (Ref. No) & 12.05 & $2.53-31.09$ & 0.001 \\
Enterococcus faecium isolated (Ref. No) & 3.37 & $1.02-11.30$ & 0.046 \\
Adjusted effect of primary study variables & & & 0.14 \\
$\quad$ Polymicrobial infection (Ref. Monobacterial) & 1.88 & $0.82-4.34$ & 0.70 \\
$\quad$ Intermediate or low susceptibility of gram-negatives to antibacterials & 1.18 & $0.51-2.70$ \\
$\quad$ Ref. High susceptibility) & & & 0.78 \\
Adjusted effect of secondary study variables & & & 0.66 \\
$\quad$ Escherichia coli isolated (Ref. No) & 1.11 & $0.52-2.50$ & 0.55 \\
$\quad$ Enterococcus faecalis isolated (Ref. No) & 0.85 & $0.41-1.78$ \\
$\quad$ Vintage (Ref. infection 1990-2006) & 0.79 & $0.36-1.72$ & 0.55 \\
& & & \\
\hline
\end{tabular}

$\mathrm{OR}=$ odds ratio $; \mathrm{CI}=$ confidence interval $\mathrm{PD}=$ peritoneal dialysis.

First-order interaction terms not significant.

\section{DISCUSSION}

According to the results of our study, peritoneal infections caused by intestinal microorganisms resulted in significant rates of mortality, permanent drop-out to hemodialysis, and, in general, treatment failure. On the other hand, PEntP was associated with higher morbidity and mortality rates than MEntP. However, a detailed analysis of our data revealed that the adverse outcome of these infections was primarily associated with the isolation of specific microorganisms, namely anaerobic bacteria and Enterococcus faecium, while the single-organism or polymicrobial nature of the infections appeared to be consequential only in terms of peritonitis-related mortality. To our knowledge, this is the first study attempting to enclose the full spectrum of PD-related peritonitis by enteric microorganisms, as opposed to previous studies, which focused separately on infections by gram-negatives $(5,6)$, Enterobacteriaceae (1), enterococci (7-9), or polymicrobial (enteric and non-enteric) peritonitis $(4,5)$.

Relatively few studies have addressed the significance of peritoneal infections by gram-negative bacteria in PD patients. Bunke et al. (6) compared 136 such episodes (after excluding infections by nonfermenting gram-negatives), with 530 cases of peritonitis by gram-positives (including Enterococcus spp.). Gram-negatives were associated with a more severe prognosis than gram-positives, a difference which was particularly marked in the absence of a simultaneous catheter infection. Szeto et al. (1) investigated 210 episodes of monobacterial peritonitis by Enterobacteriaceae (including 37 catheterdependent episodes), confirming a severe prognosis for these infections. This study disclosed a high incidence of resistance to antibacterials in these cases, particularly in patients previously treated with antibiotics. Two more recent reports from the ANZDATA registry have come to similar conclusions $(5,10)$. At least 2 studies investigating the risk profile for catheter removal due to peritonitis identified isolation of Enterobacteriaceae as an independent predictor of this outcome $(11,12)$. Isolation of multiresistant strains of gram-negatives, including those producing extended beta-lactamases, may further complicate the management and outcome of these infections (13).

Between 2 and $6 \%$ of the episodes of PD-related peritonitis are caused by enterococci (14). These typically enteric microorganisms are more frequently observed in polymicrobial infections, where they may be present in as many as $45 \%$ of the episodes (7), but can also present as single-organism peritonitis $(8,9)$. Enterococci cause relatively serious infections, with significant rates of relapse, treatment failure, and even mortality (9). Species characterization may be relevant, at least regarding the 2 most common types, namely Enterococcus faecalis and Enterococcus faecium, because the latter was associated with more complicated antibiotic resistance patterns (14). In agreement with these contentions, our data indicated that isolation of Enterococcus faecium, but not Enterococcus faecalis, was a consistent predictor of treatment failure (Table 5). 
The significance of PEntP has been a subject of limited attention in the past (15). Importantly, some relevant studies on gram-negative peritonitis excluded these infections from their analyses $(1,6)$. The best information on this question appears to come from the ANZ setting $(4,5,10)$. According to data from this registry, polymicrobial infections represent $13 \%$ of the total amount of peritonitis (10), do not present a well-defined risk profile, and was associated with increased rates of hospitalization, peritoneal catheter removal, PD drop-out, and mortality (4). However, these studies did not permit a clear differentiation between infections of enteric and non-enteric origin, although the presence of non-pseudomonal gramnegative bacteria appeared to have a detrimental influence on outcomes (4). In addition, the proportion of cases with isolation of anaerobic bacteria was remarkably low in these studies $(4,10)$. The reasons for this circumstance are not clear, but may suggest some type of bias at the time of data collection. To our knowledge, only a relatively small case series (10 episodes in 6 patients) has specifically addressed the issue of PD-related peritonitis with isolation of anaerobic bacteria (16). In this study, only 3 episodes appeared to present a background of overt abdominal disease, and antibacterial therapy alone was successful in 8 cases.

Several considerations support the convenience of a comprehensive analysis of peritonitis by intestinal microorganisms. These infections seemingly share common origins and pathways of progression (microor macroscopic transmural contamination, hematogenous). This implies potentially homogeneous risk profiles and precipitating factors, a common diagnostic approach (including abdominal imaging and surgical exploration), and similar prevention and treatment strategies. The absence of this type of inclusive approach may have contributed to the paucity of advances in the prevention and management of these infections during the last decades (17). On the other hand, a significant limitation of this strategy is the possibility that, in some cases, these infections may originate from touch contamination or peritoneal catheter-related infection. This circumstance is more likely in MEntP than in PEntP, and the high risk of an adverse outcome in the presence of anaerobics observed in our study may be partly due to the higher likelihood of significant abdominal disease in these cases. Catheter-dependent cases may be reasonably screened with the help of a careful physical examination and abdominal wall ultrasound. Touch contamination is more difficult to discard, but it may be argued that presumption of an enteric origin (less favorable hypothesis) is the most conservative and sensible first-line approach to these cases.

In our study, an exploratory laparotomy was commonly indicated in the presence of PEntP with isolation of anaerobic bacteria, but much less frequently in other settings of EntP (Table 4). Moreover, surgical exploration was negative in $38.1 \%$ of the cases in which it was undertaken, including 3 cases with a high suspicion of abdominal disease. These findings support the notions that surgical abdominal disease underlies a minority of EntP, but also that taking the decision to operate represents a major challenge in clinical practice. Given the dismal prognosis of surgical events in the absence of intervention, the convenience of an exploratory laparotomy should always be considered during the course of EntP, particularly in 3 circumstances: PEntP with isolation of anaerobics, aggressive clinical presentation, or refractory clinical course (3).

According to our data, relapse was significantly more frequent in MEntP than in PEntP (Table 3), which agrees with previous reports (5). The reasons for this finding are not clear, but the possibility of a methodologic bias should be considered, because mortality and technique failure (both more frequent in $\mathrm{PEntP}$ ) represent competing risk events for relapse of infection. 
Our study presented significant limitations, including a single-center, retrospective design. The low number of multiresistant microorganisms limited the significance of our analysis for the influence of susceptibility to antibacterials on infection outcomes. Among its strengths, we emphasize that it represents a first comprehensive approach to PD-related peritonitis of enteric origin. Exclusion of primary fungal infections and abdominal catastrophes permitted a better focused, clinically oriented approach, because these subsets demand specific diagnostic and therapeutic approaches. The high quality of our database permitted a complete and rewarding analysis of the study population.

In summary, peritoneal infections by enteric microorganisms result in significant rates of treatment failure, mortality, and permanent drop-out to hemodialysis. Polymicrobial peritonitis was associated with higher morbidity and mortality rates than single-organism infections, but an adverse outcome of these infections is primarily related to the presence of specific microorganisms, namely anaerobic bacteria and Enterococcus faecium. A comprehensive approach to peritoneal infections by intestinal gram-positive, gram-negative, and anaerobic microorganisms may help to define the clinical presentation, prognosis and treatment of these fearsome complications of PD.

\section{DISCLOSURES}

The authors have no financial conflicts of interest to declare.

\section{REFERENCES}

1. Szeto CC, Chow VCY, Chow KM, Lai RWM, Chung KY, Leung CB, et al. Enterobacteriaceae peritonitis complicating peritoneal dialysis: a review of 210 consecutive cases. Kidney Int 2006; 69:1245-52.

2. Jain AK, Blake PG. Non-pseudomonal gram-negative peritonitis. Kidney Int 2006; 69:1107-9.

3. Li PKT, Szeto CC, Piraino B, Arteaga J, Fan S, Figueiredo AE, et al. ISPD peritonitis recommendations: 2016 update on prevention and treatment. Perit Dial Int 2016; 36:481-508.

4. Barraclough K, Hawley CM, McDonald SP, Brown FG, Rosman JB, Wiggins KJ, et al. Polymicrobial peritonitis in peritoneal dialysis patients in Australia: predictors, treatment and outcomes. Am J Kidney Dis 2010; 55:121-31.

5. Jarvis EM, Hawley CM, McDonald SP, Brown SG. Rosman JB, Wiggins KJ, et al. Predictors, treatment and outcomes of non-pseudomonas gramnegative peritonitis. Kidney Int 2010; 78:408-14.

6. Bunke CM, Brier ME, Golper TA. Outcome of single organism peritonitis in peritoneal dialysis: gram-negatives versus gram-positives in the Network 9 peritonitis study. Kidney Int 1997; 52:524-9.

7. Edey M, Hawley CM, McDonald SP, Brown FG, Rosman JB, Wiggins KJ, et al. Enterococcal peritonitis in Australian peritoneal dialysis patients: predictors, treatment and outcomes in 116 cases. Nephrol Dial Transplant 2010; 25:1272-8.

8. Sutherland SM, Alexander SR, Feneberg R, Schaefer F, Warady BA. Enterococcal peritonitis in children receiving peritoneal dialysis. Nephrol Dial Transplant 2010; 25:4048-54.

9. Yip T, Tse KC, Ng F, Hung I, Lam MF, Tang S, et al. Clinical course and outcomes of single-organism Enterococcus peritonitis in peritoneal dialysis patients. Perit Dial Int $2011 ; 31: 522-8$.

10. Ghali JR, Bannister KM, Brown FG, Rosman JB, Wiggins KJ, Johnson DW, et al. Microbiology and outcomes of peritonitis in Australian peritoneal dialysis patients. Perit Dial Int 2011; 31:651-62.

11. Choi P, Nemati E, Banerjee A, Preston E, Levy J, Brown E. Peritoneal dialysis catheter removal for acute peritonitis: a retrospective analysis of factors associated with catheter removal and prolonged postoperative hospitalization. Am J Kidney Dis 2004; 43:103-11. 
12. Yang CY, Chen TW, Lin YP, Lin CC, Ng YY, Yang WC, et al. Determinants of catheter losss following continuous ambulatory peritoneal dialysis peritonitis. Perit Dial Int 2008; 28:361-70.

13. Yip T, Tse KC, Lam MF, Tang S, Li FK, Choy BY, et al. Risk factors and outcomes of extended spectrum beta-lactamase-producing E. coli peritonitis in CAPD patients. Perit Dial Int 2006; 26:191-7.

14. Pérez-Fontán M, Rodríguez-Carmona A, Rodriguez-Mayo M. Enterococcal peritonitis in peritoneal dialysis patients: last name matters. Perit Dial Int 2011; 31:513-7.

15. Holley JN, Bernardini J, Piraino B. Polymicrobial peritonitis in patients on continuous peritoneal dialysis. Am J Kidney Dis 1992; 19:182-8.

16. Chao CT, Lee SY, Yang WS, Chen HW, Fang CC, Yen CJ, et al. Peritoneal dialysis peritonitis by anaerobic pathogens: a retrospective case series. BMC Nephrology 2013; $14: 111$.

17. Szeto CC, Chow KM. Gram-negative peritonitis: the Achilles heel of peritoneal dialysis? Perit Dial Int 2006; 27(Suppl 2):S267-71. 